\title{
Nachruf Prof. Dr. Christian Herfarth
}

\author{
Jürgen Debus
}

Online publiziert: 14. November 2014

(C) Springer-Verlag Berlin Heidelberg 2014

Unser Ehrenmitglied der DEGRO, Prof. Dr. Christian Herfarth, ist am 02. September $2014 \mathrm{im}$ Alter von 81 Jahren verstorben. Prof. Christian Herfarth hat den Bereich der onkologischen Chirurgie und ihre interdisziplinäre Ausstrahlung in die Radioonkologie durch viele innovative Ansätze geprägt. Besonders hervorzuheben die Arbeiten zur onkologischen Chirurgie im Gastrointestinalbereich, die sowohl klinisch interdisziplinär aufgestellt waren als auch schon zu diesem frühen Zeitpunkt von visionärem translationalem Forschungsprogrammen begleitet wurden. Die innovative Prägung der Chirurgie durch Christian Herfarth, die eine ganze Generation von Lehrstuhlinhabern und Chefärzten hervorgebracht hat, dokumentieren sich in der Übertragung von vielfältigen Leitungspositionen wie z. B. der deutschen Gesellschaft für Chirurgie, der deutschen Krebsgesellschaft und der European Conference on Clinical Oncology sowie zahlreichen Ehrenmitgliedschaften in internationalen wissenschaftlichen Gesellschaften.

Die fast 30-jährige Tätigkeit zum Ordinarius in der Chirurgie, davon über 20 Jahre auf dem Heidelberger Lehr- stuhl, stand allgemein sein lebenslanges Streben nach der kausalen Analyse von Tumorerkrankungen, nach objektiven Kriterien für das eigene Handeln nach der Abstraktion in der Alltagsroutine nach nie erlahmender Innovation und charismatischer Motivation der jüngeren Chirurgengeneration. Hervorzuheben ist die Einführung der intraoperativen Strahlentherapie in enger Zusammenarbeit mit der Radioonkologie.

Für Christian Herfarth war immer klar, dass Chirurgen die molekularbiologische Seite ihrer Arbeit verstehen müssen. Zugleich plädierte er dafür, dass die Vertreter von Chirurgie, innerer Medizin und Strahlentherapie frühzeitig an einem Tisch zusammen arbeiten müssen, um ein interdisziplinäres Herangehen zu bedenken.

Zusammenfassend gilt er als einer der bedeutendsten Mediziner seiner Generation, der mit sehr großem persönlichem Engagement viel bewegt hat und dabei stets das Wohl der Patienten im Blick behielt.

Jürgen Debus, Heidelberg
J. Debus $(\bowtie)$

Radioonkologie und Strahlentherapie,

Universitätsklinikum Heidelberg,

Heidelberg, Deutschland

E-Mail: Juergen.Debus@med.uni-heidelberg.de 